\title{
A classification of minimal translation surfaces in Minkowski space
}

\author{
Dan Yang ${ }^{\mathrm{a}}$, Wei Dan ${ }^{\mathrm{b}, \mathrm{c}}$, Yu Fu ${ }^{\mathrm{d}, *}$ \\ a School of Mathematics, Liaoning University, Shenyang, P. R. China. \\ ${ }^{b}$ School of Mathematics and Statistic, Guangdong University of Finance and Economics, Guangzhou, P. R. China. \\ ${ }^{c}$ Faculty of Arts and Sciences, Shenzhen Technology University, Shenzhen, P. R. China. \\ ${ }^{d}$ School of Mathematics, Dongbei University of Finance and Economics, Dalian, P. R. China.
}

Communicated by D. Baleanu

\begin{abstract}
Minimal surfaces are well known as a class of surfaces with vanishing mean curvature which minimize area within a given boundary configuration since 19th century. This fact was implicitly proved by Lagrange for nonparametric surfaces in 1760 , and then by Meusnier in 1776 who used the analytic expression for the mean curvature. Mathematically, a minimal surface corresponds to the solution of a nonlinear partial differential equation. By solving some differential equations, in this paper we give a complete and explicit classification of minimal translation surfaces in an n-dimensional Minkowski space.
\end{abstract}

Keywords: Minimal surfaces, translation surfaces, Minkowski space.

2010 MSC: 53A10.

(C)2018 All rights reserved.

\section{Introduction}

Minimal surfaces are among the most natural objects in differential geometry, and have been studied during the last two and half centuries since J. L. Lagrange. In particular, minimal surfaces have encountered striking applications in other fields, like mathematical physics, conformal geometry, computer aided design, among others. Even though it has been the subject of intense activity, many basic problems remain open, for instance, the complete classification of minimal surfaces in 3-dimensional space. In general, to consider the solutions of some differential equations is essential and important, for instance, [5, 7-10, 12]. In order to search for more minimal surfaces, some natural geometric assumptions arise. In the 3-dimensional Euclidean space $\mathbb{E}^{3}$, a surface is called a translation surface if it is given by an immersion

$$
r: U \subset \mathbb{E}^{2} \rightarrow \mathbb{E}^{3}:(x, y) \rightarrow(x, y, z),
$$

where $z=f(x)+g(y), f(x)$ and $g(y)$ are differentiable functions. The first study of translation surfaces

\footnotetext{
*Corresponding author

Email addresses: dlutyangdan@126.com (Dan Yang), wdan@gdufe.edu.cn (Wei Dan), fuyumath@163.com (Yu Fu)
}

doi: $10.22436 /$ jnsa.011.03.12

Received: 2017-07-06 Revised: 2017-12-31 Accepted: 2018-01-07 
is due to Scherk, in 1835 who proved that, besides the plane, the only minimal translation surface is the so-called Scherk surface given by

$$
z=\frac{1}{a} \ln \left|\frac{\cos (a x)}{\cos (a y)}\right|,
$$

where $a$ is a nonzero constant. In 1991, the minimal translation surfaces were generalized to minimal translation hypersurfaces by F. Dillen, L. Verstraelen and G. Zafindratafa [4]. Since then, translation surfaces (hypersurfaces) have been investigated intensively by geometers (see $[1,6,7,10,11]$ ).

In 1994, L. Verstraelen, J. Walrave and S. Yaprak studied minimal translation surfaces in Euclidean space $\mathbb{E}^{n}$ for arbitrary codimension by proving that, besides the plane, the only minimal translation surface in $\mathbb{E}^{n}$ is given by

$$
r(s, t)=\left(s, t, \frac{c_{3}}{m} \ln \left|\frac{\cos (\sqrt{m} s)}{\cos (\sqrt{m} t)}\right|, \cdots, \frac{c_{n}}{m} \ln \left|\frac{\cos (\sqrt{m} s)}{\cos (\sqrt{m} t)}\right|\right),
$$

where $m=\sum_{k=3}^{n} c_{k}^{2}$ and $c_{k}$ are constant, see details in [13].

In n-dimensional Minkowski space $\mathbb{E}_{1}^{\mathfrak{n}}$, there are two types of translation surfaces as follows (c.f. $[5,7]):$

$$
\begin{aligned}
& \text { I. } r(s, t)=\left(s, t, x_{3}(s, t), x_{4}(s, t), \cdots, x_{n}(s, t)\right), \\
& \text { II. } r(s, t)=\left(x_{3}(s, t), x_{4}(s, t), \cdots, x_{n}(s, t), s, t\right),
\end{aligned}
$$

where $x_{i}(s, t)=f_{i}(s)+g_{i}(t), 3 \leqslant i \leqslant n$. The problem to classify minimal translation surfaces in an n-dimensional Minkowski space becomes interesting and important. Generally, the classification problems for submanifolds in Minkowski space are more complicated than the Euclidean case because of the indefiniteness of the metric. In this paper, we will deal with the similar problem for translation surfaces as L. Verstraelen et al.'s work [13] in Minkowski space $\mathbb{E}_{1}^{n}$. We obtain a complete classification of minimal translation surfaces with type I and type II, respectively, in the n-dimensional Minkowski space $\mathbb{E}_{1}^{n}$. Specifically, we get the following classification theorems.

Theorem 1.1. Let $M$ be a translation surface with type I in Minkowski space $\mathbb{E}_{1}^{n}$. Then $M$ is minimal if and only if $M$ is a part of the following:

1. a plane;

2. $\mathrm{r}(\mathrm{s}, \mathrm{t})=\mathrm{p}(\mathrm{s})+\mathrm{q}(\mathrm{t})$, where $\mathrm{p}(\mathrm{s})$ and $\mathrm{q}(\mathrm{t})$ are lightlike vectors in Minkowski space $\mathbb{E}_{1}^{\mathrm{n}}$;

3. a flat B-scroll over a null curve;

4. $r(s, t)=\left(s, t, \frac{c_{3}}{2}\left(t^{2}-s^{2}\right), \cdots, \frac{c_{n}}{2}\left(t^{2}-s^{2}\right)\right)$;

5. $r(s, t)=\left(s, t, \frac{c_{3}}{m} \ln \left|\frac{\cos (\sqrt{m} s)}{\cos (\sqrt{m} t)}\right|, \cdots, \frac{c_{n}}{m} \ln \left|\frac{\cos (\sqrt{m} s)}{\cos (\sqrt{m} t)}\right|\right)$ for $m>0$;

6. $r(s, t)=\left(s, t, \frac{c_{3}}{m} \ln \left|\frac{\sinh (\sqrt{-m} s)}{\sinh (\sqrt{-m} t)}\right|, \cdots, \frac{c_{n}}{m} \ln \left|\frac{\sinh (\sqrt{-m} s)}{\sinh (\sqrt{-m} t)}\right|\right)$ for $m<0$;

7. $r(s, t)=\left(s, t, \frac{c_{3}}{m} \ln \left|\frac{\sinh (\sqrt{-m} s)}{\cosh (\sqrt{-m} t)}\right|, \cdots, \frac{c_{n}}{m} \ln \left|\frac{\sinh (\sqrt{-m} s)}{\cosh (\sqrt{-m} t)}\right|\right)$ for $m<0$;

8. $r(s, t)=\left(s, t, \frac{c_{3}}{m} \ln \left|\frac{\cosh (\sqrt{-m} s)}{\cosh (\sqrt{-m} t)}\right|, \cdots, \frac{c_{n}}{m} \ln \left|\frac{\cosh (\sqrt{-m} s)}{\cosh (\sqrt{-m} t)}\right|\right)$ for $m<0$,

where $\mathrm{m}=\mathrm{c}_{3}^{2}+\cdots+\mathrm{c}_{\mathrm{n}-1}^{2}-\mathrm{c}_{\mathrm{n}}^{2}$ is constant.

Theorem 1.2. Let $M$ be a translation surface with type II in Minkowski space $\mathbb{E}_{1}^{n}$. Then $M$ is minimal if and only if $M$ is a part of the following:

1. a plane;

2. a flat B-scroll over a null curve;

3. $r(s, t)=\left(\frac{c_{3}}{m} \ln \left|\frac{\cosh (\sqrt{m} t)}{\cos (\sqrt{m} s)}\right|, \cdots, \frac{c_{n}}{m} \ln \left|\frac{\cosh (\sqrt{m} t)}{\cos (\sqrt{m} s)}\right|, s, t\right)$;

4. $r(s, t)=\left(\frac{c_{3}}{m} \ln \left|\frac{\sinh (\sqrt{m} t)}{\cos (\sqrt{m} s)}\right|, \cdots, \frac{c_{n}}{m} \ln \left|\frac{\sinh (\sqrt{m} t)}{\cos (\sqrt{m} s)}\right|, s, t\right)$,

where $\mathrm{m}=\mathrm{c}_{3}^{2}+\cdots+\mathrm{c}_{\mathrm{n}}^{2}$ is a positive constant. 
Remark 1.3. For $n=3, M$ is a minimal translation surface (spacelike or timelike) in Minkowski space $\mathbb{E}_{1}^{3}$. In this case, Theorem 1.1 and Theorem 1.2 are generalizations of the 3-dimensional case (see [5, 7] for details).

Remark 1.4. Noting that $\mathrm{n}>3$ in the cases (4) and (5) in Theorem 1.1, there is no corresponding minimal translation surface in Minkowski 3-space $\mathbb{E}_{1}^{3}([5])$.

\section{Preliminaries}

Let $\mathbb{E}_{1}^{\mathfrak{n}}$ be $\mathrm{n}$-dimensional Minkowski space with the metric

$$
<,>=d x_{1}^{2}+d x_{2}^{2}+\cdots+d x_{n-1}^{2}-d x_{n}^{2} .
$$

We denote $M$ by a surface immersed in Minkowski space $\mathbb{E}_{1}^{n}$ parameterized by

$$
r: U \subset \mathbb{E}^{2} \rightarrow \mathbb{E}^{3}:(s, t) \rightarrow\left(x_{1}(s, t), x_{2}(s, t), \cdots, x_{n}(s, t)\right) .
$$

Then the components of the first fundamental form for surface $M$ are given by

$$
g_{11}=<r_{s}, r_{s}>=r_{s} \cdot r_{s}, \quad g_{12}=<r_{s}, r_{t}>=r_{s} \cdot r_{t}, \quad g_{22}=<r_{t}, r_{t}>=r_{t} \cdot r_{t} .
$$

A surface $M$ is spacelike if the induced metric on surface $M$ is positive, that is $g_{11} g_{22}-g_{12}^{2}>0$; a surface $M$ is timelike if the induced metric on surface $M$ is indefinite, that is, $g_{11} g_{22}-g_{12}^{2}<0$, respectively.

Let $\left\{\eta_{3}, \cdots, \eta_{n}\right\}$ be normal frame field of the surface $M$ immersed in Minkowski space $\mathbb{E}_{1}^{n}$. Then the components of the second fundamental form concerning the normal vector $\eta_{k}$ are given by

$$
\begin{aligned}
& b_{11}\left(\eta_{k}\right)=<r_{s s}, \eta_{k}>=r_{s s} \cdot \eta_{k} \\
& b_{12}\left(\eta_{k}\right)=<r_{s t}, \eta_{k}>=r_{s t} \cdot \eta_{k}, \\
& b_{22}\left(\eta_{k}\right)=<r_{t t}, \eta_{k}>=r_{t t} \cdot \eta_{k} .
\end{aligned}
$$

Recall that the component of the mean curvature vector $H_{\eta_{k}}$ is given by (c.f. $[2,3]$ )

$$
H_{\eta_{k}}=\frac{g_{22} b_{11}+g_{11} b_{22}-2 g_{12} b_{12}}{2\left(g_{11} g_{22}-g_{12}^{2}\right)} \text {. }
$$

Hence a surface $M$ is minimal (or maximal for a spacelike surface) if and only if $H_{\eta_{k}}=0$ for all $k=$ $3, \ldots, n$.

\section{A proof of Theorem 1.1}

In this section, by considering some differential equations we will give the concrete parameterizations of translation surfaces with type (I).

First of all, it is easy to check that

$$
\begin{aligned}
& r_{s}=\left(1,0, f_{3}^{\prime}(s), f_{4}^{\prime}(s), \cdots, f_{n}^{\prime}(s)\right), \\
& r_{t}=\left(0,1, g_{3}^{\prime}(t), g_{4}^{\prime}(t), \cdots, g_{n}^{\prime}(t)\right) .
\end{aligned}
$$

We could build the normal frame fields in the normal space as follows

$$
\begin{aligned}
\eta_{3} & =\left(-f_{3}^{\prime}(s),-g_{3}^{\prime}(t), 1,0, \cdots, 0,0\right), \\
& \vdots \\
\eta_{n-1} & =\left(-f_{n-1}^{\prime}(s),-g_{\mathfrak{n}-1}^{\prime}(t), 0,0, \cdots, 1,0\right), \\
\eta_{\mathfrak{n}} & =\left(f_{\mathfrak{n}}^{\prime}(s), g_{\mathfrak{n}}^{\prime}(t), 0,0, \cdots, 0,1\right) .
\end{aligned}
$$


It is easy to check that the components of the first fundamental form are in the following forms

$$
\begin{aligned}
& g_{11}=r_{s} \cdot r_{s}=1+f_{3}^{\prime 2}+\cdots+f_{n-1}^{\prime 2}-f_{n}^{\prime 2}, \\
& g_{22}=r_{t} \cdot r_{t}=1+g_{3}^{\prime 2}+\cdots+g_{n-1}^{\prime 2}-g_{n}^{\prime 2}, \\
& g_{12}=r_{s} \cdot r_{t}=f_{3}^{\prime} g_{3}^{\prime}+\cdots+f_{n-1}^{\prime} g_{n-1}^{\prime}-f_{n}^{\prime} g_{n}^{\prime},
\end{aligned}
$$

and the components of the second fundamental form are given by

$$
\begin{aligned}
& b_{11}\left(\eta_{k}\right)=r_{s s} \cdot \eta_{k}=f_{k}^{\prime \prime}, \quad b_{12}\left(\eta_{k}\right)=r_{s t} \cdot \eta_{k}=0, \quad b_{22}\left(\eta_{k}\right)=r_{t t} \cdot \eta_{k}=g_{k}^{\prime \prime} \\
& b_{11}\left(\eta_{n}\right)=r_{s s} \cdot \eta_{n}=-f_{n}^{\prime \prime}, \quad b_{12}\left(\eta_{n}\right)=r_{s t} \cdot \eta_{n}=0, \quad b_{22}\left(\eta_{n}\right)=r_{t t} \cdot \eta_{n}=-g_{n}^{\prime \prime}
\end{aligned}
$$

for $\mathrm{k}=3, \cdots, \mathrm{n}-1$.

Let $H\left(\eta_{i}\right)$ be the mean curvature with respect to the normal vector $\eta_{i}$. Then the surface $M$ is minimal if and only if $\mathrm{H}\left(\eta_{i}\right)=0(i=3,4, \cdots, n)$, that is,

$$
\left[1+g_{3}^{\prime 2}+\cdots+g_{n-1}^{\prime 2}-g_{n}^{\prime 2}\right] f_{k}^{\prime \prime}+\left[1+f_{3}^{\prime 2}+\cdots+f_{n-1}^{\prime 2}-f_{n}^{\prime 2}\right] g_{k}^{\prime \prime}=0
$$

We first consider the case $1+g_{3}^{\prime 2}+\cdots+g_{n-1}^{\prime 2}-g_{n}^{\prime 2}=1+f_{3}^{\prime 2}+\cdots+f_{n-1}^{\prime 2}-f_{n}^{\prime 2}=0$. If we let

$$
p(s)=\left(s, 0, f_{3}, \cdots, f_{n}\right), \quad q(t)=\left(0, t, g_{3}, \cdots, g_{n}\right),
$$

then both $p(s)$ and $q(t)$ are two lightlike vectors in Minkowski space $\mathbb{E}_{1}^{n}$. So the case (2) in Theorem 1.1 is obtained.

If $1+g_{3}^{\prime 2}+\cdots+g_{n-1}^{\prime 2}-g_{n}^{\prime 2}=0$ and $1+f_{3}^{\prime 2}+\cdots+f_{n-1}^{\prime 2}-f_{n}^{\prime 2} \neq 0$, by (3.2) we have that $g_{k}^{\prime \prime}=0$, so $g_{k}=c_{k} t$ for $k=3, \cdots, n$. Then the surface $M$ takes the form

$$
r(s, t)=\left(s, 0, f_{3}, \cdots, f_{n}\right)+t\left(0,1, c_{3}, \cdots, c_{n}\right),
$$

which implies that $M$ is a flat B-scroll over a null curve. Hence, we obtain the case (3) in Theorem 1.1.

If $1+g_{3}^{\prime 2}+\cdots+g_{n-1}^{\prime 2}-g_{n}^{\prime 2} \neq 0$ and $1+f_{3}^{\prime 2}+\cdots+f_{n-1}^{\prime 2}-f_{n}^{\prime 2}=0$, similarly we obtain the case (3) in Theorem 1.1.

We now consider the case $1+g_{3}^{\prime 2}+\cdots+g_{n-1}^{\prime 2}-g_{n}^{\prime 2} \neq 0$ and $1+f_{3}^{\prime 2}+\cdots+f_{n-1}^{\prime 2}-f_{n}^{\prime 2} \neq 0$. From (3.2), there exist constants $c_{k}$ such that

$$
-\frac{f_{k}^{\prime \prime}}{1+f_{3}^{\prime 2}+\cdots+f_{n-1}^{\prime 2}-f_{n}^{\prime 2}}=\frac{g_{k}^{\prime \prime}}{1+g_{3}^{\prime 2}+\cdots+g_{n-1}^{\prime 2}-g_{n}^{\prime 2}}=c_{k}
$$

for $k=3, \cdots, n$. Hence

$$
\begin{aligned}
& f_{k}^{\prime \prime}=-c_{k}\left[1+f_{3}^{\prime 2}+\cdots+f_{n-1}^{\prime 2}-f_{n}^{\prime 2}\right], \\
& g_{k}^{\prime \prime}=c_{k}\left[1+g_{3}^{\prime 2}+\cdots+g_{n-1}^{\prime 2}-g_{n}^{\prime 2}\right] .
\end{aligned}
$$

If $c_{k}=0$, then from (3.4) and (3.5) we conclude that $f_{k}(s)=a_{k} s+b_{k}$, and $g_{k}(t)=c_{k} t+d_{k}$ for $k=$ $3, \cdots, n$. Thus, $M$ is a plane in the Minkowski space $\mathbb{E}_{1}^{n}$, which gives the case (1) in Theorem 1.1.

Now suppose that at least one of the $c_{k} \neq 0$. By re-arranging the indices (if necessary) we can assume that $c_{3} \neq 0$. Then taking the ratio of the equation given in (3.5), we have

$$
\frac{f_{k}^{\prime \prime}}{f_{3}^{\prime \prime}}=\frac{g_{k}^{\prime \prime}}{g_{3}^{\prime \prime}}=\frac{c_{k}}{c_{3}}, \quad k=3, \cdots, n
$$

Hence

$$
f_{k}^{\prime \prime}(s)=\frac{c_{k}}{c_{3}} f_{3}^{\prime \prime}(s), \quad g_{k}^{\prime \prime}(t)=\frac{c_{k}}{c_{3}} g_{3}^{\prime \prime}(t), \quad k=3, \cdots, n .
$$


After a translation of the variable s, we get

$$
f_{k}^{\prime}(s)=\frac{c_{k}}{c_{3}} f_{3}^{\prime}(s), \quad g_{k}^{\prime}(t)=\frac{c_{k}}{c_{3}} g_{3}^{\prime}(t), \quad k=3, \cdots, n .
$$

Putting $k=3$ in (3.5), we have

$$
\begin{aligned}
f_{3}^{\prime \prime} & =-c_{3}\left[1+f_{3}^{\prime 2}+\cdots+f_{n-1}^{\prime 2}-f_{n}^{\prime 2}\right], \\
g_{3}^{\prime \prime} & =c_{3}\left[1+g_{3}^{\prime 2}+\cdots+g_{n-1}^{\prime 2}-g_{n}^{\prime 2}\right] .
\end{aligned}
$$

Substituting (3.7) into (3.8) and (3.9), we have

$$
f_{3}^{\prime \prime}=-\frac{m}{c_{3}}\left(f_{3}^{\prime}\right)^{2}-c_{3}, \quad g_{3}^{\prime \prime}=\frac{m}{c_{3}}\left(g_{3}^{\prime}\right)^{2}+c_{3},
$$

where $m=c_{3}^{2}+\cdots+c_{n-1}^{2}-c_{n}^{2}$.

We now distinguish the following three cases:

Case 1. $m=0$. In this case, by (3.10) we have

$$
f_{3}^{\prime \prime}=-c_{3}, \quad g_{3}^{\prime \prime}=c_{3},
$$

and hence

$$
f_{3}^{\prime}=-c_{3} s+d_{1}, \quad g_{3}^{\prime}=c_{3} t+d_{2} .
$$

It follows from (3.7) that

$$
f_{k}^{\prime}=-c_{k} s+\frac{c_{k}}{c_{3}} d_{1}, \quad g_{k}^{\prime}=c_{k} t+\frac{c_{k}}{c_{3}} d_{2} .
$$

By integration, and after an affine transformation, we have

$$
f_{k}=-\frac{c_{k}}{2} s^{2}, \quad g_{k}=\frac{c_{k}}{2} t^{2} .
$$

So the case (4) in Theorem 1.1 is obtained.

Case 2. $m>0$. In this case, from (3.10) we have

$$
\frac{m}{c_{3}} f_{3}^{\prime \prime}=-\left[\left(\frac{m}{c_{3}} f_{3}^{\prime}\right)^{2}+m\right], \quad \frac{m}{c_{3}} g_{3}^{\prime \prime}=\left(\frac{m}{c_{3}} g_{3}^{\prime}\right)^{2}+m .
$$

By solving the equation (3.14), we have

$$
f_{3}^{\prime}=-\frac{c_{3}}{\sqrt{m}} \tan (\sqrt{m} s), \quad g_{3}^{\prime}=\frac{c_{3}}{\sqrt{m}} \tan (\sqrt{m} t) .
$$

From (3.7), we have

$$
f_{k}^{\prime}=-\frac{c_{k}}{\sqrt{m}} \tan (\sqrt{m} s), \quad g_{k}^{\prime}=\frac{c_{k}}{\sqrt{m}} \tan (\sqrt{m} t),
$$

and hence

$$
f_{k}=\frac{c_{k}}{m} \ln |\cos (\sqrt{m} s)|, \quad g_{k}=-\frac{c_{k}}{m} \ln |\cos (\sqrt{m} t)|,
$$

which gives the case (5) in Theorem 1.1.

Case 3. $\mathrm{m}<0$. In this case from (3.10) we have

$$
\frac{m}{c_{3}} f_{3}^{\prime \prime}=-\left[\left(\frac{m}{c_{3}} f_{3}^{\prime}\right)^{2}-(\sqrt{-m})^{2}\right], \quad \frac{m}{c_{3}} g_{3}^{\prime \prime}=\left(\frac{m}{c_{3}} g_{3}^{\prime}\right)^{2}-(\sqrt{-m})^{2} .
$$


By solving the first equation of (3.16), we have

$$
f_{3}^{\prime}=-\frac{c_{3}}{\sqrt{-m}} \operatorname{coth}(\sqrt{-m} s) \quad \text { or } \quad f_{3}^{\prime}=-\frac{c_{3}}{\sqrt{-m}} \tanh (\sqrt{-m} s),
$$

which together with (3.7) gives

$$
f_{k}^{\prime}=-\frac{c_{k}}{\sqrt{-m}} \operatorname{coth}(\sqrt{-m} s) \quad \text { or } \quad f_{k}^{\prime}=-\frac{c_{k}}{\sqrt{-m}} \tanh (\sqrt{-m} s),
$$

and hence

$$
f_{k}=\frac{c_{k}}{m} \ln |\sinh (\sqrt{-m} s)| \quad \text { or } \quad f_{k}=\frac{c_{k}}{m} \ln |\cosh (\sqrt{-m} s)| .
$$

Similarly, by the second equation of (3.16), we have

$$
g_{k}=-\frac{c_{k}}{m} \ln |\sinh (\sqrt{-m} t)| \quad \text { or } \quad g_{k}=-\frac{c_{k}}{m} \ln |\cosh (\sqrt{-m} t)| .
$$

Combining (3.18) and (3.19), the cases (6), (7) and (8) in Theorem 1.1 are obtained. This completes the proof of Theorem 1.1.

\section{A proof of Theorem 1.2}

In this section, we consider the translation surfaces with type (II). Similar to the discussion on the proof of Theorem 1.1, we give the normal vector field

$$
\begin{aligned}
\eta_{3} & =\left(1,0, \cdots,-f_{3}^{\prime}(s), g_{3}^{\prime}(t)\right), \\
& \vdots \\
\eta_{\mathfrak{n}} & =\left(0,0, \cdots, 1,-f_{\mathfrak{n}}^{\prime}(s), g_{\mathfrak{n}}^{\prime}(t)\right) .
\end{aligned}
$$

The components of the first fundamental form and the second fundamental form are written respectively as

$$
g_{11}=f_{3}^{\prime 2}+\cdots+f_{n}^{\prime 2}+1, \quad g_{22}=g_{3}^{\prime 2}+\cdots+g_{n}^{\prime 2}-1, \quad g_{12}=f_{3}^{\prime} g_{3}^{\prime}+\cdots+f_{n}^{\prime} g_{n}^{\prime},
$$

and

$$
b_{11}\left(\eta_{k}\right)=f_{k}^{\prime \prime}, \quad b_{12}\left(\eta_{k}\right)=0, \quad b_{22}\left(\eta_{k}\right)=g_{k}^{\prime \prime}
$$

for $\mathrm{k}=3, \cdots, \mathrm{n}$.

Hence, it is easy to check that the surface $M$ is minimal if and only if

$$
\left[g_{3}^{\prime 2}+\cdots+g_{n}^{\prime 2}-1\right] f_{k}^{\prime \prime}+\left[f_{3}^{\prime 2}+\cdots+f_{n}^{\prime 2}+1\right] g_{k}^{\prime \prime}=0
$$

If $g_{3}^{\prime 2}+\cdots+g_{n}^{\prime 2}-1=0$, by (4.1) we have that $g_{k}^{\prime \prime}=0$. So $g(k)=c_{k} t$ for $k=3, \cdots, n$. Then the surface $M$ is given by

$$
r(s, t)=\left(f_{3}, \cdots, f_{n}, s, 0\right)+t\left(c_{3}, \cdots, c_{n}, 0,1\right),
$$

which implies that $M$ is a flat B-scroll over a null curve. So the case (2) in Theorem 1.2 is given.

If $g_{3}^{\prime 2}+\cdots+g_{n}^{\prime 2}-1 \neq 0$, from (4.1), there exist constants $c_{k}$ such that

$$
\frac{f_{k}^{\prime \prime}}{f_{3}^{\prime 2}+\cdots+f_{n}^{\prime 2}+1}=-\frac{g_{k}^{\prime \prime}}{g_{3}^{\prime 2}+\cdots+g_{n}^{\prime 2}-1}=c_{k}
$$


for $k=3, \cdots, n$. Hence

$$
f_{k}^{\prime \prime}=c_{k}\left[f_{3}^{\prime 2}+\cdots+f_{n}^{\prime 2}+1\right], \quad g_{k}^{\prime \prime}=-c_{k}\left[g_{3}^{\prime 2}+\cdots+g_{n}^{\prime 2}-1\right]
$$

If $c_{k}=0$, then from (4.3) we conclude that $f_{k}(s)=a_{k} s+b_{k}, g_{k}(t)=c_{k} t+d_{k}$ for $k=3, \cdots, n$. Thus $M$ is a plane in the Minkowski space $\mathbb{E}_{1}^{n}$ and the case (1) in Theorem 1.2 is obtained.

Now suppose that at least one of the $c_{k} \neq 0$, we also assume that $c_{3} \neq 0$. Similar to the discussion of the surfaces of type (I), we have

$$
f_{k}^{\prime}(s)=\frac{c_{k}}{c_{3}} f_{3}^{\prime}(s), \quad g_{k}^{\prime}(t)=\frac{c_{k}}{c_{3}} g_{3}^{\prime}(t), \quad k=3, \cdots, n .
$$

Substituting (4.4) into (4.3) for $k=3$, we have

$$
f_{3}^{\prime \prime}=\frac{m}{c_{3}}\left(f_{3}^{\prime}\right)^{2}+c_{3}, \quad g_{3}^{\prime \prime}=-\frac{m}{c_{3}}\left(g_{3}^{\prime}\right)^{2}+c_{3},
$$

where $m=c_{3}^{2}+\cdots+c_{n}^{2}>0$. By solving the first equation of (4.5), we have

$$
f_{3}^{\prime}=\frac{c_{3}}{\sqrt{m}} \tan (\sqrt{m} s)
$$

which together with (4.4) gives

$$
f_{k}^{\prime}=\frac{c_{k}}{\sqrt{m}} \tan (\sqrt{m} s)
$$

and hence

$$
f_{k}=-\frac{c_{k}}{m} \ln |\cos (\sqrt{m} s)| .
$$

Similarly, by the second equation of (4.5), we have

$$
g_{k}=\frac{c_{k}}{m} \ln |\cosh (\sqrt{m} t)|, \quad \text { or } \quad g_{k}=\frac{c_{k}}{m} \ln |\sinh (\sqrt{m} t)| .
$$

So the cases (3) and (4) in Theorem 1.2 are obtained. This completes the proof of Theorem 1.2.

\section{Acknowledgment}

The authors were supported by NSFC (No. 11601068, No. 11701098), the Liaoning Science and Technology Project (No. 201602528) and Guangdong Science Foundation 2015A030310304.

\section{References}

[1] A. Bueno, R. López, Translation surfaces of linear Weingarten type, arXive, 2014 (2014), 7 pages. 1

[2] B.-Y. Chen, Geometry of Submanifolds, Marcel Dekker, New York, (1973). 2

[3] B.-Y. Chen, Pseudo-Riemannian Geometry, S-invariants and Applications, With a foreword by Leopold Verstraelen, World Scientific Publishing Co., Hackensack, (2011). 2

[4] F. Dillen, L. Verstraelen, G. Zafindratafa, A generalization of the translation surfaces of Scherk, Diff. Geom. in honor of Radu Rosca (KUL), (1991), 107-109. 1

[5] W. Goemans, Surfaces in three-dimensional Euclidean and Minkowski space, in particular a study of Weingarten surfaces, PhD Thesis, Katholieke Univ. Leuven, (2010). 1, 1.3, 1.4

[6] B. P. Lima, N. L. Santos, P. A. Sousa, Translation hypersurfaces with constant scalar curvature into the Euclidean space, Israel J. Math., 201 (2014), 797-811. 1

[7] H. Liu, Translation surfaces with constant mean curvature in 3-dimensional spaces, J. Geom., 64 (1999), 141-149. 1, 1.3

[8] M. Marin, On weak solutions in elasticity of dipolar bodies with voids, J. Comp. Appl. Math., 82 (1997), 291-297.

[9] M. Marin, Harmonic vibrations in thermoelasticity of microstretch materials, J. Vib. Acoust. ASME, 2010 (2010), 6 pages.

[10] M. I. Munteanu, A. I. Nistor, Polynomial Translation Weingarten Surfaces in 3-dimensional Euclidean space, Differential geometry, 316-320, World Sci. Publ., Hackensack, (2009). 1

[11] K. Seo, Translation hypersurfaces with constant curvature in space forms, Osaka J. Math., 50 (2013), 631-641. 1

[12] K. Sharma, M. Marin, Effect of distinct conductive and thermodynamic temperatures on the reflection of plane waves in micropolar elastic half-space, Politehn. Univ. Bucharest Sci. Bull. Ser. A Appl. Math. Phys., 75 (2013), 121-132. 1

[13] L. Verstraelen, J. Walrave, S. Yaprak, The minimal translation surfaces in Euclidean space, Soochow J. Math., 20 (1994), 77-82. 1 